\title{
Alternative responses of primary tumor cells and glioblastoma cell lines to N,N-bis-(8-hydroxyquinoline-5-yl methyl)-benzyl substituted amines: Cell death versus $P 53$-independent senescence
}

\author{
JEAN-LOUIS KRAUS ${ }^{1,2}$, FILIPPO CONTI ${ }^{1}$, SÉBASTIEN MADONNA ${ }^{1,2}$, \\ AURELIE TCHOGHANDJIAN ${ }^{3}$ and CHRISTOPHE BECLIN ${ }^{1}$
}

\author{
${ }^{1}$ CNRS, IBDML-UMR-6216, Campus de Luminy Case 907; ${ }^{2}$ Laboratoire de Chimie Biomoléculaire, \\ IBDML-UMR-6216-CNRS, Faculté des Sciences de Luminy, Université de la Méditerranée, 13288 Marseille cedex 09; \\ ${ }^{3}$ Université de la Méditerranée, Faculté de Médecine Timone, Laboratoire de Biopathologie \\ de l'Adhésion et de la Signalisation, Marseille F-13000, France
}

Received July 7, 2010; Accepted August 20, 2010

DOI: 10.3892/ijo_00000798

\begin{abstract}
N,N-bis-(8-hydroxyquinoline-5-yl methyl)-benzyl substituted amines (HQNBA) represent a new class of compounds showing anti-cancer activity. At the chemical level the compounds were shown to react preferentially with thiol radicals which may lead to unfolded cysteine containing proteins and subsequent ER-stress. At the molecular level, treatment of U87 cells with this class of derivatives induced an over-expression of stress genes, including P53 and numerous $P 53$ target genes. By generating shRNA U87 cell clones impaired in $P 53$ expression we found that $P 53$ mediates neither proliferation arrest of treated U87 cells nor over-expression of potential P53 targets. Moreover, we discovered that a representative HQNBA derivative (JLK1486) induces strong but transient senescence in U87 cells in a P53-independent manner. We demonstrate that, in contrast to its effect on established glioblastoma cell lines, JLK1486 induces extensive death of primary glioblastoma cells. We provide evidence that both caspase 3 , and 7 activation, and cathepsin B and D activities account for at least part of this cell death.
\end{abstract}

\section{Introduction}

We recently identified N,N-bis-(8-hydroxyquinoline-5-yl methyl)-benzyl substituted amines (HQNBA) as a new anticancer family of compounds (1) and showed that these compounds act through the formation of a quinone methide intermediate with alkylating properties. However, in contrast to most pharmaceutical alkylating compounds, quinone methide is not sufficiently electrophile to react with DNA

Correspondence to: Dr Christophe Beclin, IBDML-UMR-6216, Campus de Luminy, Case 907, 13288 Marseille cedex 09, France E-mail: christophe.beclin@ibdml.univmed.fr

Key words: glioblastoma, P53, senescence, cell death, HQNBA but is reactive enough to covalently bind adducts to strong nucleophiles such as thiol radicals (2). In addition, we demonstrated that these compounds can induce apoptotic cell death in several cancer cell lines $(1,2)$.

More recently, we found that in different glioblastoma cell lines (U87, U373, Hs683, T989G) HQNBA derivatives efficiently blocked cell proliferation and migration in the absence of apopotosis (2). Taking advantage of the ability of U87 cells to survive these treatments, we analyzed the cellular response at the molecular level. For this purpose micro-array analysis on U87 cells treated vs non-treated with active HQNBA derivative was performed and led to the identification of several families of genes whose expression changed in response to treatment. We observed an extensive induction of stress genes, in particular of genes specific to ER-stress and UPR response, as a consequence of HQNBA treatment. This induction is likely due to the formation of adducts on thiol radicals, leading to the unfolding of cysteine containg proteins. In parallel, inflammation and apoptotic genes were found to be induced. Concomitantly to this stress induction we also observed a clear signature of cell cycle inhibition, in accordance with the phenotype of treated cells (2).

$P 53$ is a transcription factor considered as the major mediator of stress response. P53 activity can be regulated both at the transcriptional and at the post-translational levels (3) and mediate several cell pathways leading to apoptosis, cell cycle arrest, autophagy and senescence (3).

For these reasons P53 has been called the cellular 'gatekeeper' (4) or the guardian of the genome (5) and is often found mutated in cancer cells (6). In U87 cells, however, P53 exists in a non-mutated form and has been involved in the response of these cells to anti-cancer compounds, in particular the DNA alkylating agent Temozolomide, the main chemical arm against glioma $(7,8)$, and also to nitrosoureas (9). Moreover, in our micro-array analyses of HQNBA-treated U87 cells we detected up-regulation of P53 and several genes of its signaling pathway (2).

We investigated the involvement of P53 in the response to JLK1486, a representative HQNBA derivative. U87 cell 
clones knocked-down for P53 exhibited the same cell cycle arrest in response to JLK1486 treatment. Moreover, several P53 targets were similarly induced by JLK1486 in knockeddown and control clones. From these results we could conclude that $P 53$ is not a major mediator of the response of U87 cells towards JLK1486 treatment.

In addition, we show that JLK1486 treatment induced a clear P53-independent senescence phenotype in U87 cells, and that this senescence is transitory allowing cells to re-start proliferating after drug removal. Finally, we demonstrated that JLK1486-treated primary glioblastoma cell lines behaved differently from U87, as they underwent extensive cell death in the absence of signs of senescence.

\section{Materials and methods}

Cell culture, transfection, nucleic acid, in vitro survival/ proliferation assay, chemicals. The glioblastoma cell line U87gfp has been described (2). U87gfp cells and U87gfpderived cells were cultured in MEM medium (Invitrogen) supplemented with $10 \%$ FBS. U87gfp cells stably expressing shRNA vectors were established after FuGene 6 (Roche) transfection followed by selection with $1 \mathrm{mg} / \mathrm{ml}$ of puromycin. To generate U87gfp cell clones knocked down for P53 we used two P53 targeting shRNA vectors: pPURU6P53-1 vector (10) and pCL-SI-MSCV puro-H1R-P53-Ri vector (11). The non-targeting control shRNA vector was pPURU6 (10). The clones expressing pPURU6-P53-1, pCLSI-MSCVpuro-H1R-P53-Ri and pPURU6 were labeled Dx, Ax or Ex and Bx, respectively.

siRNA were transfected in U87gfp cells by nucleofection (Lonza) as follows. One million U87gfp cells and $50 \mu \mathrm{mol}$ of siRNA were mixed in a cuvette and submitted to nucleofection. After nucleofection, cells were re-suspended in MEM FBS $10 \%$ and cultivated for $24 \mathrm{~h}$ before being trypsinized and seeded in 96-wells plate for proliferation assay (2). siRNA were provided by Dharmacon (now part of Thermo Scientific). For targeting ATF4 we used the SMART-pool targeting human ATF4 and for the non-relevant siRNA we used the SMARTpool targeting mouse $C X C R 4$.

Primary glioblastoma cells were amplified as neurospheres as described (21). For survival/proliferation assay, primary cells were cultivated in same neurosphere medium on polyDL-ornithin coated wells of a 96-well plate (21). Cells were counted after labeling of living cells with 45 min incubation at $37^{\circ} \mathrm{C}$ in $4.1 \mu \mathrm{g} / \mathrm{ml}$ calcein AM (VWR, France). Chemicals were added 1 day after cell plating. Z-VAD(OMe)-FMK [ZVal-Ala-DL-Asp(OMe)-fluoromethylketone] was obtained from Bachem (Switzerland), the cathepsin B inhibitor CA074Me was obtained from Calbiochem. For proliferation assay, the initial time-point was the time at which chemicals were added. Synthesis of HQNBA derivatives and related spectroscopic data have been previously reported $(1,2)$.

$q R T-P C R$. qRT-PCR was performed using the One Step MesaGreen qPCR Mastermix for SYBR assay kit (Eurogenetech) on a $\mathrm{iQ}^{\mathrm{TM}} 5$ Real-Time PCR detection system (BioRad). Statitical analysis of the results was achieved using the joined software. For RNA extraction cells were trypsinized and RNA was immediately extracted using RNeasy mini kit

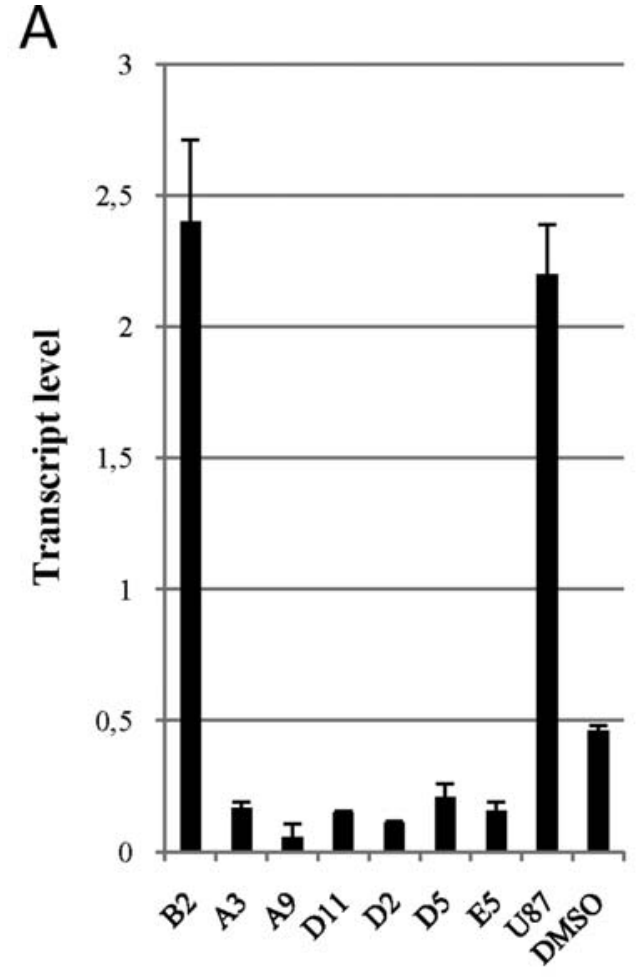

B

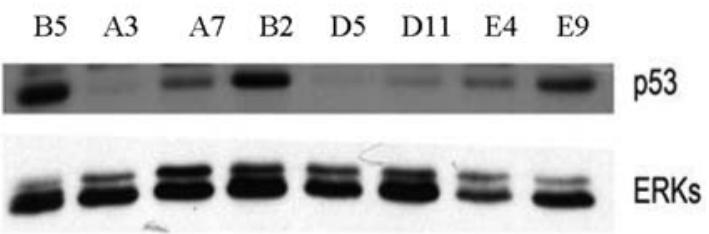

Figure 1. Generation of U87-derived cell clones knocked down for P53. P53 expression level in the U87-derived shRNA clones was analysed by qRT-PCR (A) and Western blotting (B). Clones A3, A7, A9 E4, E5 and E9 express pCL-SI-MSCVpuro-H1R-P53-Ri (11), clones D2, D5, D11 express pPURU6-P53-1 (10). B2 and B4 express the control vector pPURU6 (10). All samples were treated $24 \mathrm{~h}$ with JLK1486 0.5 $\mu \mathrm{M}$, except sample DMSO issued from DMSO-treated U87 cells.

(Qiagen). To evaluate the effect of JLK1486 on gene expression, RNA was extracted $24 \mathrm{~h}$ after JLK1486 supplementation. The sequence of primers used were: For P53 detection, GCG CACAGAGGAAGAGAATC and CAAGGCCTCATTCAG CTCTC; for P21 detection, GAGCGATGGAACTTCGACTT and CAGGTCCACATGGTCTTCCT; for ATF4 detection, TCAAACCTCATGGGTTCTCC and AGGTCATCTGGCA TGGTTTC; for Chop detection, TGGAAGCCTGGTATGA GGAC and TGTGACCTCTGCTGGTTCTG; for SESN2 detection, GCTCATCACCAAGGAACACA and AGCCAA ACACGAAGGAGGA.

Western blotting, senescence and caspase activation kits, histochemistry. Western blots were performed on cell extracts prepared in EBM buffer [EBM 2X: $20 \mathrm{mM}$ Tris- $\mathrm{HCl}(\mathrm{pH} 7.5)$, $150 \mathrm{mM} \mathrm{NaCl}, 1 \%$ Triton, $10 \%$ glycerol, $5 \mathrm{mM}$ EDTA, $5 \mathrm{mM}$ EGTA, $1 \mathrm{mM}$ phenylmethylsulfonyl fluoride, $10 \mathrm{mM} \mathrm{NaF}$, $1 \mathrm{mM}$ NaPP, $1 \mathrm{mM}$ orthovanadate, $10 \mathrm{mM}$ b-glycerophosphate, $5 \mu \mathrm{g} / \mathrm{ml}$ leupeptin and pepstatin, $5 \mathrm{mM}$ benzamidine, 
A

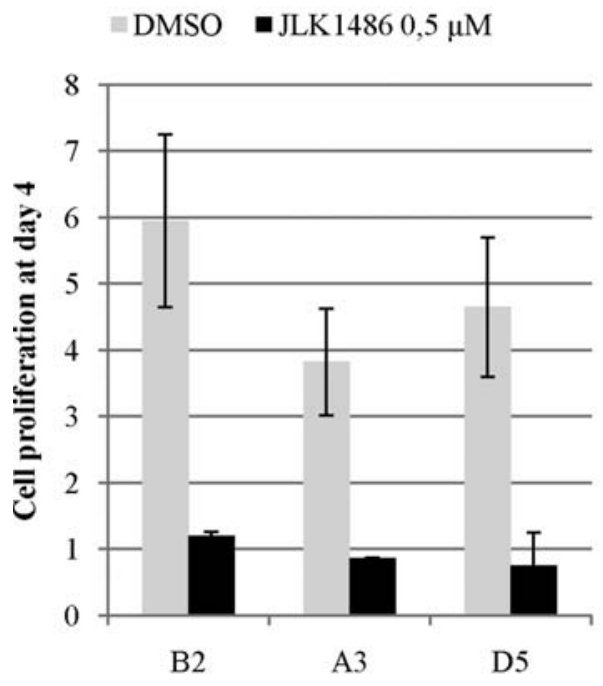

B
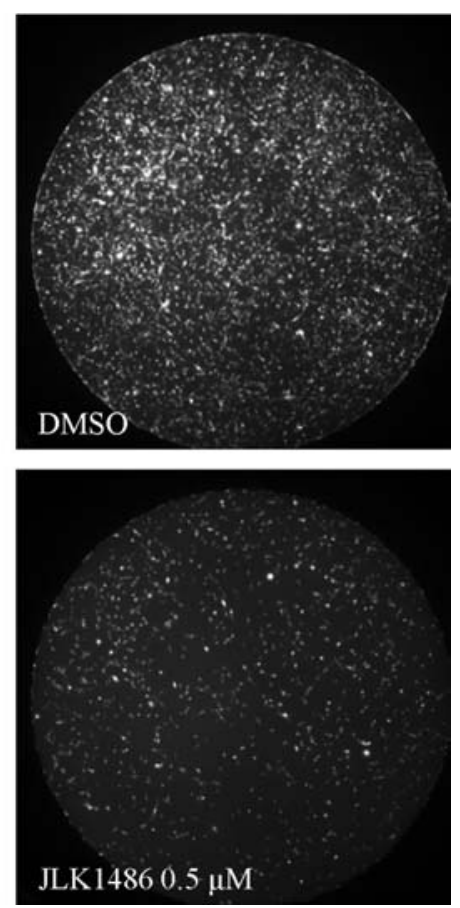

Figure 2. Knock-down of P53 does not change the response of U87 cells to JLK1486 treatment. (A) Control cell clones B2 and shP53 clones A3, D5 and E11 were treated with DMSO or JLK1486 $0.5 \mu \mathrm{M}$ and their proliferation rate at day 4 was quantified. (B) Images taken by the Flash-cytometer representing the whole well of 96-wells plate containing cells from shRNA clone A3, 4 days after compound supplementation.

$2 \mu \mathrm{g} / \mathrm{ml}$ aprotinin]. For immunoblotting, $25 \mu \mathrm{g}$ of protein extract were separated by SDS-PAGE, blotted onto nitrocellulose membrane and detected with specific antibodies. All immunoblots were revealed by ECL plus (Amersham).

Senescence in cells was detected using the Senescence Cells Histochemical Staining kit (Sigma) following manufacturer's recommendations. Caspase 3/7 activation was quantified using Apo-ONE homogeneous caspase $3 / 7$ assay kit (Promega). The antibodies against cathepsin D (human Ab-2), P53 (Pab240) and ERK (ref. 9102) were provided from Calbiochem, Santa Cruz (Santa Cruz, CA) and Cell Signaling, respectively.

\section{Results}

Generation of cell clones expressing shP53 vectors. We generated U87-derived cell clones stably expressing shRNAs targeting P53 using two different constructs, pPURU6-P53-1 and pCL-SI-MSCV puro-H1R-P53-Ri, previously described in (10) and (11), respectively. P53 expression was analyzed by qRT-PCR and Western blotting. Several clones showed a reduction in $P 53$ expression by more than $90 \%$ both at the mRNA and protein level (Fig. 1). These knocked-down clones obtained with both shP53 vectors were used for all subsequent experiments.

When shP53 clones were treated with JLK1486 cells exhibited the same cell cycle inhibition as cells expressing the control vector (Fig. 2). Moreover, similarly to control clones, $P 53$-reduced cells also fully survived to JLK1486. This result suggests that the cellular response to JLK1486 treatment is independent of $P 53$. Alternatively, the remaining P53 expression in the knocked-down clones may still be sufficient to support mediation of a stress response. To address this possibility we analyzed in the knocked-down and control clones the expression of several genes known to be regulated through $P 53$. We chose two direct targets of $P 53$, $P 21$ (4) and SESN2 (12,13). Furthermore, one gene involved in the UPR response which has already been described as regulated by $P 53$, Chop (14) was selected. In our micro-array experiments, we found strong expression of SESN2 and Chop after $24 \mathrm{~h}$ of treatment (x14 and 15, respectively) whereas P21 exhibited a mild activation of 2.3 -fold (2). We confirmed by qRT-PCR that these genes are specifically induced by JLK1486 and that they were expressed at the same level as non-treated cells when U87 cells were challenged with the non-bioactive HQNBA derivatives JLK1525, JLK1540 and JLK1576 (2) (Fig. 3A).

As expected, $P 21$ showed a strong reduction in expression in the two analyzed knock-down clones, showing that in this situation P53 cannot sustain normal P21 expression (Fig. 3B). However, in the same clones SESN2 and Chop are expressed at comparable levels as in control clones, either in non-treated or in JLK1486-treated conditions, suggesting that P53 is not responsible for activation of these genes. A P53-independent activation has already been reported for Chop (15) but to our knowledge not for SESN2 (Fig. 3B).

JLK1486 blocks proliferation and migration through senescence induction independently on P53 expression. KB3 cells undergo apoptosis when treated with HQNBA derivatives at nanomolar concentrations (1). However, in glioblastoma cell lines, such as U87, U373, Hs683 or T989G (2), no cell death was observed, despite strong induction of apoptotic genes, even at a concentration of $20 \mu \mathrm{M}$. Instead, when the concentration in JLK1486 reaches $0.2 \mu \mathrm{M}$, complete cell cycle and migration arrest were observed (2). When we treated the shP53 clones 
A

chop $\mathbf{m} 53$ =SESN2 $=\mathrm{P} 21$

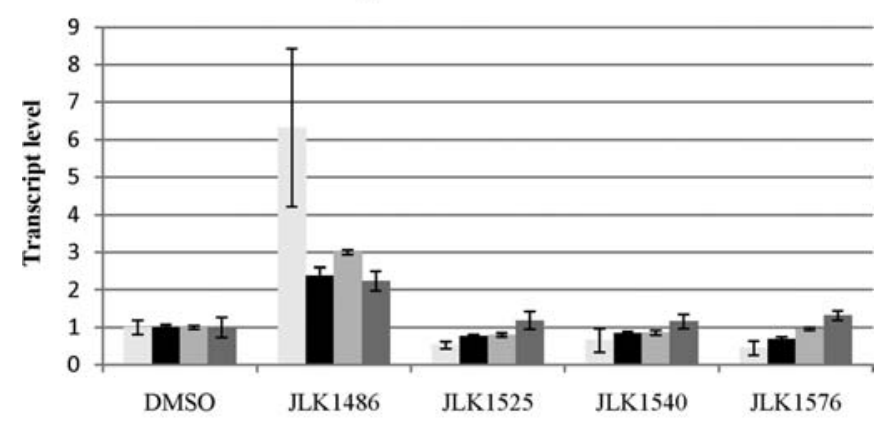

B

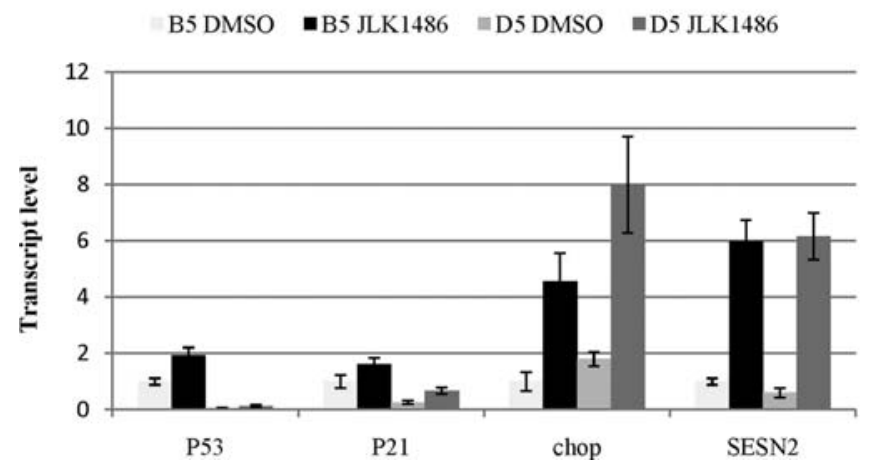

Figure 3. The expression level of P53 and the potential P53 targets correlate with bio-activity of bis-8-hydroxiquinoleine compounds but the activity of the compounds is not mediated by P53. (A) qRT-PCR analysis of U87 cells treated with DMSO or $0.5 \mu \mathrm{M}$ of the bio-active bis-8hydroxiquinoleine compound JLK1486 or $2 \mu \mathrm{M}$ of the non-active bis-8hydroxiquinoleine compounds JLK1525, JLK1540 and JLK1576. (B) qRTPCR analysis of P53, P21, Chop or SESN2 expression level in cell clones B5 (expressing control vector) or D5 (knocked-down for P53) treated with DMSO or $0.5 \mu \mathrm{M}$ JLK1486.

with JLK1486 we observed a similar proliferation and migration blockade without visible cell death, comparable to control cells (Fig. 3A). Moreover, the product concentration at which maximum effect is reached in shP53 clones and in control cells is identical. This shows that P53 does not mediate the proliferation and migration inhibition induced by JLK1486.

Senescence is a cytostatic process triggered by a variety of insults. The process is considered as an anti-tumor mechanism, as it is generally found to be irreversible and has been shown to participate in vivo to tumor clearance. At the opposite, in glioblastoma cell lines radiotherapy induced senescence followed by senescence exit has been proposed to allow tumor cells to resist apoptosis (16). We checked for senescence induction in treated cells. Using an assay based on B-Gal staining, we observed that JLK1486-induced cell cycle arrest is accompanied by triggering of senescence (Fig. 4A). After 3 days of treatment we removed the drug and followed the culture for several days. We observed senescence exit in several wells as soon as 7 days after drug withdrawal (Fig. 4B) showing that senescence is not irreversible in U87-derived cells treated with JLK1486. These experiments showed that senescence is extensively but transiently induced by JLK1486 and may account for cell proliferation inhibition in U87.

In vivo restoration of $P 53$ function allowed the authors to implicate P53 in the triggering of senescence in several models of tumors (17-19). We thus investigated the senescence triggering in shP53 clones after JLK1486 treatment. We showed that, similarly to that observed in our proliferation and migration assays, shP53 clones senesced at a comparable rate as control clones when treated with JLK1486 (Fig. 4A). Also, senescence exit can be observed one week after drug withdrawal in shP53 cells containing wells (data not shown). These results show that P53 is involved neither in the induction nor the exit of JLK1486-mediated senescence in U87 cells.

An alternative pathway in senescence induction pathway involving $P 21, P 27$ and ATF4 as key factors, has been recently identified (20). In our micro-array analysis we detected strong induction of ATF4 transcription (about 4-fold) in U87 cells treated with JLK1486. This transcription activation occurred only with active compounds and did not depend on P53 expression (data not shown). We thus checked the involvement of ATF4 in the response of U87 cells towards JLK1486. Transfection of ATF4 targeting siRNA in U87 cells led to strong ATF4 inhibition (Fig. 4C), while transfected U87 cells exhibited the same inhibition in proliferation when treated with JLK1486 as cells transfected with control siRNA (Fig. 4D). Altogether, these data suggest that the senescence pathway induced in U87 cells by JLK1486 treatment is independent of P53 expression and does not seem to require proper ATF4 expression.

JLK1486 induces cell death in primary glioblastoma cells. We recently described the establishment and characterization of a primary glioblastoma cell line GBM6 that exhibits features reminiscent of the clinical characteristics of the original tumor in vitro culture and orthotopic grafting experiments (21). These cells are maintained and grown in culture as neurospheres in FBS-free medium supplemented with EGF and FGF. Such primary cancer cell lines are supposed to be more relevant for in vitro studies than traditional established cancer cell lines cultivated in medium with high FBS content.

We applied our proliferation assay on GBM6 cells treated with JLK1486 and observed that the cells exhibited sensitivity to the compound at the same concentration range as U87 cells. However, in contrast to U87 cells, in GBM6 cells JLK1486 induced progressive but extensive cell death (Fig. 5A). When we checked for senescence induction in cells that have not yet undergone cell death, B-Gal staining was similar in treated and non-treated cells (data not shown). This result suggests that glioblastoma cells challenged with HQNBA derivatives can respond by two alternative ways: senescence induction or cell death.

We studied the cell death pathway induced in JLK1486treated GBM6 cells and found that cell death shared classical features of apoptotic cell death as, for example, strong caspase 3/7 induction (Fig. 5B) and nucleus fragmentation (Fig. 5C). Moreover, application of the caspase $3 / 7$ inhibitor Z-VAD [Z-VAD(OMe)-FMK] rescued part of the JLK1486-induced cell death (Fig. 5A). However, the fact that Z-VAD-mediated rescue is only partial, even at very high concentration $(100 \mu \mathrm{M})$ (Fig. 5A), suggests that JLK1486-mediated GBM6 cell death relies on an alternative pathway. The observation that treated GBM6 exhibited frequent cathepsin D (Fig. 5C) release before dying indicated that lysosomal stress can account for at least part of the observed cell death. Indeed, it is known that lyso- 
A
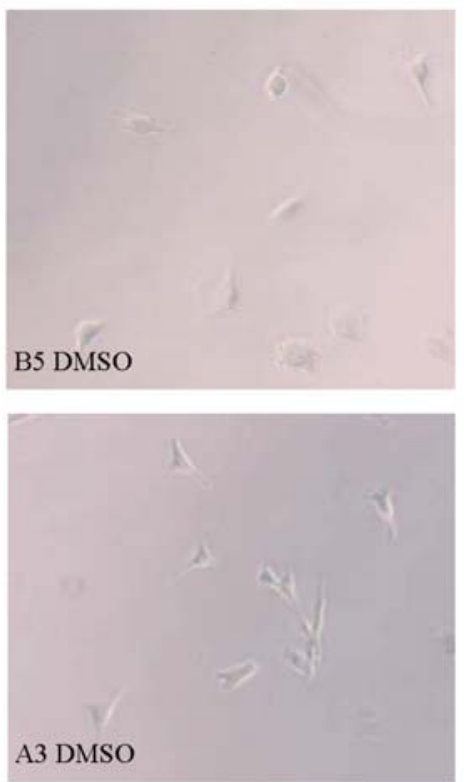

A3 DMSO
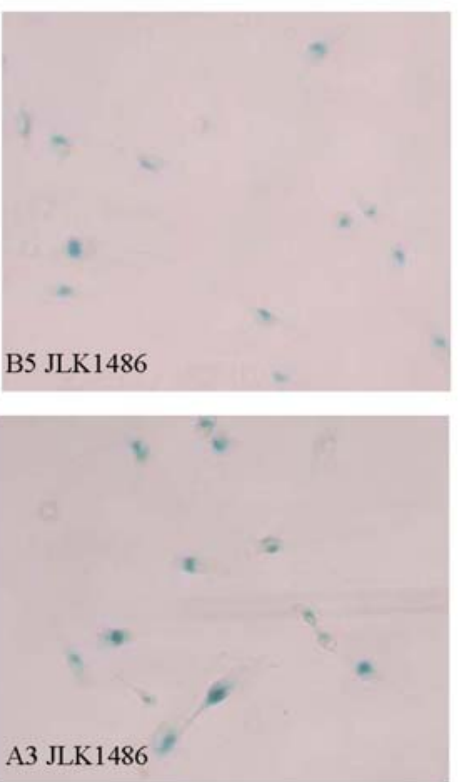

B
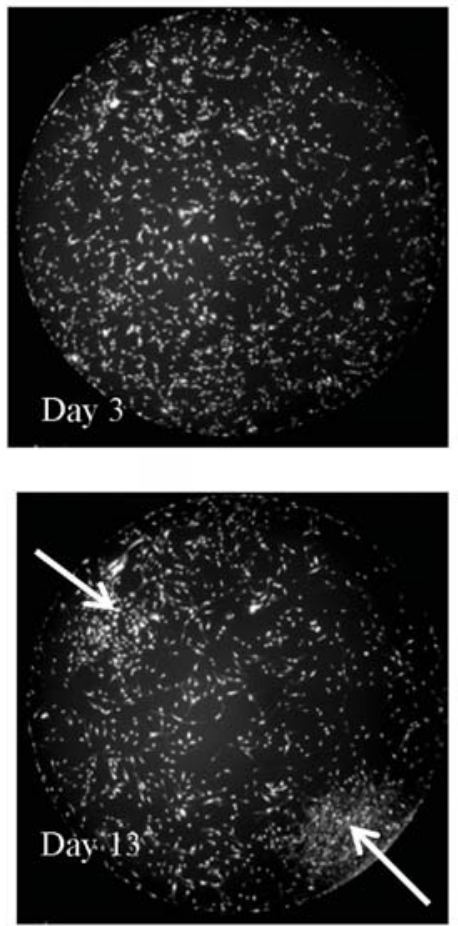

C

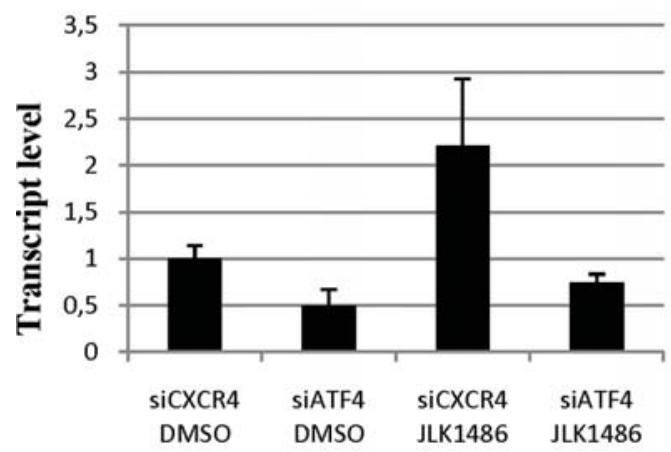

D

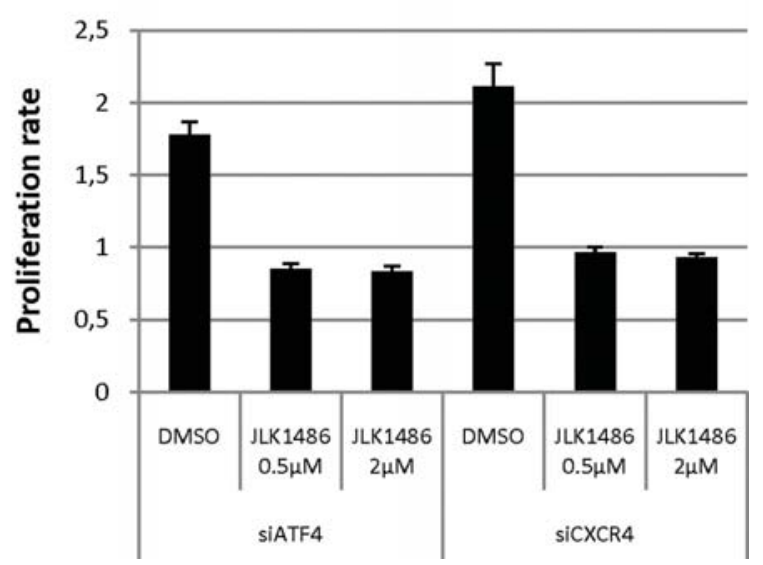

Figure 4. JLK1486 treatment induces a P53- and ATF4-independent transient senescence. (A) Senescence assay on clones B5 (expressing control vector) or A3 (knocked-down for P53) treated for 3 days with DMSO or $0.5 \mu \mathrm{M}$ JLK1486. (B) Images taken by the Flash-cytometer representing the same well containing U87 cells at 2 time-points. Cells were treated for 3 days with JLK1486 $0.5 \mu \mathrm{M}$. At day 3 medium was changed with a drug-free medium and cells were kept in culture for 10 more days. The 2 arrows show cells that have re-started to proliferate. (C) qRT-PCR of nucleofected U87 cells transfected by mouse $C X C R 4$ targeting siRNA or ATF4 targeting siRNA, after $24 \mathrm{~h}$ treatment with DMSO or $0.5 \mu \mathrm{M}$ JLK1486. (D) Proliferation rate at day 3 of U87 cells transfected with siATF4 or siCXCR4 and treated with DMSO or $0.5 \mu \mathrm{M}$ JLK1486 or $2 \mu \mathrm{M}$ JLK1486.

somal stress may lead to cell death through the liberation of proteases like cathepsin B and D in the cytoplasm of proteases (22). We evaluated the effect of chemical inhibitors of both cathepsins on JLK1486-mediated GBM6 cell death. We treated GBM6 cells with JLK1486 together with compound CA074-Me, a commercially available cathepsin B inhibitor, and/or a naphtyl birayl piperazine derivative with aspartylprotease inhibitory property; compound 4c (23). We observed that both inhibitors synergistically acted to rescue JLK1486-mediated GBM6 cell death (Fig. 5D). However as for Z-VAD activity, the rescue is only partial, suggesting either that other degrading enzymes are released after lysosomal stress or that other mechanism than lysosomal stress is involved in the induction of cell death.

\section{Discussion}

Glioblastomas are the most aggressive brain cancers, remaining reluctant to most therapies so far. Among the reasons invoked to explain such extreme resistance to treatments are the very high invasiveness of tumour cells, impairing its complete neurosurgical resection, and the resistance of tumour cells to growth inhibition and apoptosis (24). The only, modestly successful, approach has been defined as maximum surgical 
A

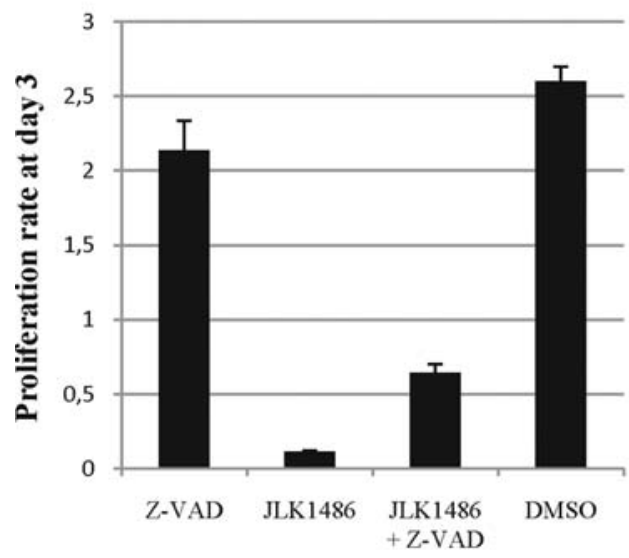

C

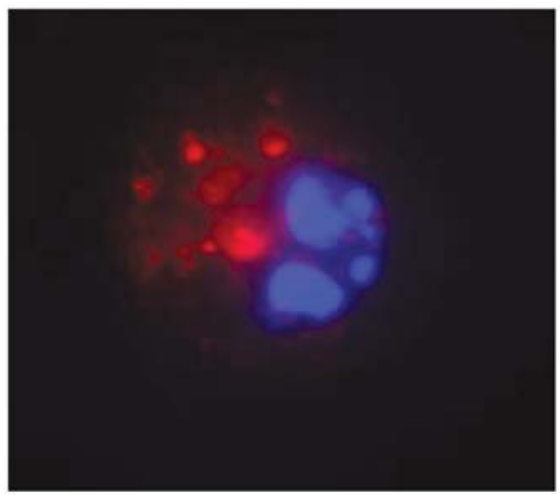

B

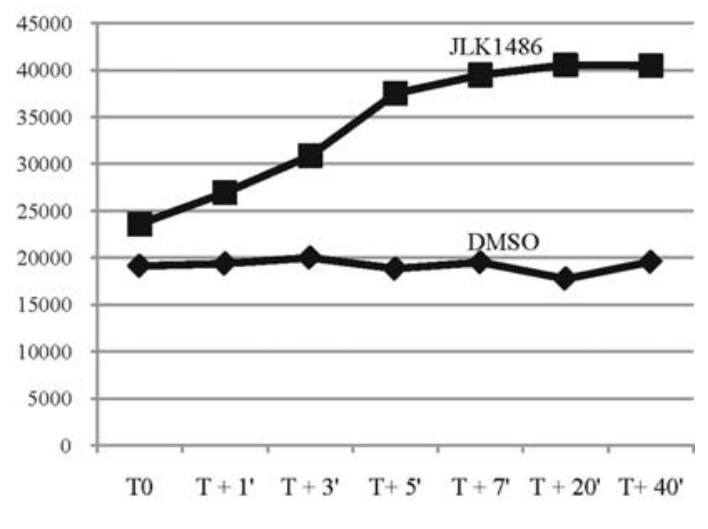

D

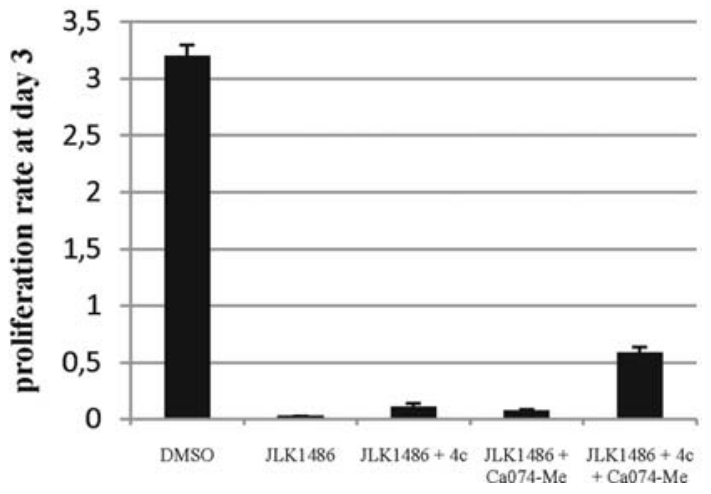

Figure 5. JLK1486 provokes extensive cell death in GBM6 cells that shows features of conventional apoptotic cell death and that partly depends on cathepsin $\mathrm{B}$ and D activities. (A) Proliferation rate at day 3 of U87 cells treated with DMSO or treated with $0.5 \mu \mathrm{M}$ JLK1486 and/or $100 \mu \mathrm{M}$ Z-VAD. (B) Measurement of caspase 3, 7 activity at successive time-points after addition of the caspase substrate in culture wells containing GBM6 cells treated 3 days with DMSO or JLK1486. (C) Confocal image of GBM6 cells treated 3 days with JLK1486 and labeled with DAPI (blue) or cathepsin D antibody (red). (D) Proliferate rate of GBM6 cells treated either with DMSO or with JLK1486 or with a combination of JLK1486 with cathepsin B inhibitor (Ca074-Me) and/or cathepsin D inhibitor (4c).

resection followed by radiotherapy in combination with Temozolomide-based chemotherapy $(25,26)$. Temozolomide is a DNA alkylating agent with activity in several glioblastoma cell lines shown to induce autophagy and increase the lysosomal volume (27). However, the use of Temozolomide has a limited clinical outcome and its activity is dependent on the methylation status of the O6-methylguanine-DNA methyltransferase (MGMT) promoter. The development of new treatments therefore constitutes a major goal in GBM patient therapy.

We demonstrate here that glioblastoma cells show sensitivity towards HQNBA derivatives. However, as for other anticancer compounds, the molecule acts at a concentration range higher than for other cancer cell lines (2). Furthermore, we show that cell growth and migration arrest of U87 cells treated with JLK1486, the reference bio-active HQNBA derivative, is accompanied by strong senescence induction.

This senescence induction, while cytostatic, is accompanied by complete survival. Moreover, this senescence is only transitory allowing cells to re-start proliferating after drug removal, a mechanism that might well underlie drug resistance of tumor cells. It would be interesting to see if there is a causative link between senescence and cell survival. In this case senescence could be considered as a drug resistance mechanism allowing cells to evade cell death and would thus represent a pathway to target in order to potentiate anti-cancer drugs.

We have started to investigate the molecular mechanism involved in this process. Several senescence-inducing pathways have been described, the principal being the P19ArflP53 axis. As P53 has been involved in Temozolomide induction of senescence in U87 cells (28) and because our gene expression analysis showed over-expression of P53 and P53 targets (2), we addressed the involvement of P53 in the response of U87 cells to HQNBA derivatives. First, we found that over-expression of $P 53$ and a set of several potential P53 targets specifically occurred exclusively in the presence of bio-active HQNBA derivatives but not when HQNBA 
analogs belonging to the same chemical series but lacking activity on cell proliferation and migration were used. However, extensive inhibition of P53 expression did not modify the response of U87 to bis-8-hydroxiquinoleine treatment. Moreover, the different P53 targets we investigated were still induced by JLK1486 in the shP53 clones, at the same level for Chop and SESN2 but at lower level for $P 21$. These results strongly suggest that the participation of $P 53$ in the mediation of stress response observed in treated U87 cells is at best minor.

The P16/Rb axis (29) and the P21/P27/ATF4 system (20) represent alternative routes for senescence. However, our work suggests that neither pathway is involved in the senescence induced in U87 cells treated with JLK1486. First, our microarray analysis did not show significant change in P16Ink4a expression upon HQNBA treatment (2). Second, ATF4 knockdown did not modify the phenotype of treated U87 cells. Altogether these results indicate that the senescence induced in U87 cells treated with HQNBA derivatives involves a new and non-conventional pathway.

GBM6 cells responded differently than U87 to JLK1486 treatment as they underwent extensive cell death and did not show senescence induction. This response to the drug did not appear specific to the GBM6 line as another primary glioblastoma cell line, GBM9 (21), similarly exhibited cell death when treated with JLK1486 (data not shown). Our experimental data obtained with GBM6 cells allow relying this cell death to several cellular pathways.

First we provided chemical and molecular evidence that HQNBA derivatives were able to induce strong ER-stress (2) and showed that these compounds can cause global protein thiol redox modifications. The subsequent unfolded-cysteine containing proteins are supposed to be retained in the ER triggering ER-stress (30). The UPR response to ER-stress leads to expression of chaperones, translation lowering and misfolded protein degradation (31). UPR may thus be considered as a survival pathway and may account for survival of JLK1486-treated U87 cells. When ER-stress exceeds a specific threshold, which depends on the cell type, cells are committed to death (32). This mechanism may thus be the initial step towards cell death in GBM6 cells.

Second, microscopic analysis showed frequent cathepsin D release in the treated GBM6 cells. Moreover application of cathepsin inhibitors rescued part of the induced cell death. These results strongly suggest an involvement of lysosomal stress, including lysosome membrane permeabilisation and protease (cathepsins) release in the cytoplasm, in GBM6 cell death. Third we demonstrated that caspase 3/7 were activated in treated GBM6 cells and that Z-VAD supplementation allowed partial cell death reversion, demonstrating the ability of HQNBA derivatives to induce of a typical type-I apoptosis.

Several reports showed that these pathways may be connected pairwise in injured cells. ER-stress in glioblastoma cells has been shown able to induce caspase-dependent apoptosis $(33,34)$. ER-stress and lysosome-related cell death has been functionally connected in glioblastoma cells (35). Finally lysosomal stress may lead to caspase-dependent apoptotic cell death as well as necrotic or autophagic cell death in a P53-dependent or -independent manner $(22,36,37)$. The fact that Z-VAD as well as cathepsin inhibitors can only rescue part of cell death indicates that each individual event cannot account for the amount of cell death we observed. This implies that the cascade of cellular events leading to the death of GBM6 cells is probably not linear and should take several alternative routes towards cell death.

An important observation in this study is that primary and established cell lines respond very differently towards HQNBA derivatives treatment. While GBM6 cells react by activating a strong cell death response, an observation that holds promise for the future therapeutic use of this class of compounds, U87 cells enter senescence when treated with JLK1486 and subsequently fully survived to treatment. The discrepancy between behaviors of established versus primary cell lines in response to HQNBA treatment may reflect a real variability between different glioblastoma cell types with two alternative responses existing in this type of cancers. However, an alternative explanation for this difference may originate from the very artificial conditions in which the established cell lines, such as U87, were cultivated and maintained for long time. In this case our results may lead to the consideration that the use of such cell lines is not appropriate for analyzing the response of glioblastoma cells to anti-cancer drugs.

\section{Acknowledgements}

This study was supported by the 'Institut National du Cancer (INCA), Canceropole Provence Alpes Cote d'azur (PACA)'. The authors thank Harold Cremer for critical reading of the manuscript. We thank Dr Fukunaga and Dr Kiyono for providing with $P 53$ targeting shRNA vectors.

\section{References}

1. Moret V, Laras Y, Cresteil T, Aubert G, Ping DQ, Di C, Barthélémy-Requin M, Beclin C, Peyrot V, Allegro D, Rolland A, De Angelis F, Gatti E, Pierre P, Pasquini L, Petrucci E, Testa U and Kraus JL: Discovery of a new family of bis-8-hydroxyquinoline substituted benzylamines with pro-apoptotic activity in cancer cells: synthesis, structure-activity relationship, and action mechanism studies. Eur J Med Chem 44: 558-567, 2009.

2. Madonna S, Beclin C, Laras Y, Moret V, Marcowycz A, Lamoral-Theys D, Dubois J, Barthelemy-Requin M, Lenglet G, Depauw S, Cresteil T, Aubert G, Monnier V, Kiss R, DavidCordonnier MH and Kraus JL: Structure-activity relationships and mechanism of action of antitumor bis 8-hydroxyquinoline substituted benzylamines. Eur J Med Chem 45: 623-638, 2010.

3. Millau JF, Bastien N and Drouin R: P53 transcriptional activities: a general overview and some thoughts. Mutat Res 681: 118-133, 2009.

4. Levine AJ: P53, the cellular gatekeeper for growth and division. Cell 88: 323-331, 1997.

5. Lane DP: P53, guardian of the genome. Nature 359: 486-487, 1992.

6. Olivier M, Eeles R, Hollstein M, Khan MA, Harris CC and Hainaut P: The IARC TP53 database: new online mutation analysis and recommendations to users. Hum Mutat 19: 607-614, 2002.

7. Hermisson M, Klumpp A, Wick W, Wischhusen J, Nagel G, Roos W, Kaina B and Weller M: O6-methylguanine DNA methyltransferase and P53 status predict temozolomide sensitivity in human malignant glioma cells. J Neurochem 96: 766-776, 2006.

8. Roos WP, Batista LF, Naumann SC, Wick W, Weller M, Menck CF and Kaina B: Apoptosis in malignant glioma cells triggered by the temozolomide-induced DNA lesion O6methylguanine. Oncogene 26: 186-197, 2007.

9. Batista LF, Roos WP, Christmann M, Menck CF and Kaina B: Differential sensitivity of malignant glioma cells to methylating and chloroethylating anticancer drugs: P53 determines the switch by regulating xpc, ddb2, and DNA double-strand breaks. Cancer Res 67: 11886-11895, 2007. 
10. Watanabe-Fukunaga R, Iida S, Shimizu Y, Nagata S and Fukunaga R: SEI family of nuclear factors regulates P53dependent transcriptional activation. Genes Cells 10: 851-860, 2005.

11. Yugawa T, Handa K, Narisawa-Saito M, Ohno S, Fujita M and Kiyono T: Regulation of Notch1 gene expression by P53 in epithelial cells. Mol Cell Biol 27: 3732-3742, 2007.

12. Budanov AV, Shoshani T, Faerman A, Zelin E, Kamer I, Kalinski H, Gorodin S, Fishman A, Chajut A, Einat P, Skaliter R, Gudkov AV, Chumakov PM and Feinstein E: Identification of a novel stress-responsive gene Hi95 involved in regulation of cell viability. Oncogene 21: 6017-6031, 2002.

13. Maiuri MC, Malik SA, Morselli E, Kepp O, Criollo A, Mouchel PL, Carnuccio R and Kroemer G: Stimulation of autophagy by the P53 target gene Sestrin2. Cell Cycle 8: $1571-1576,2009$.

14. Liu T, Laurell C, Selivanova G, Lundeberg J, Nilsson P and Wiman KG: Hypoxia induces P53-dependent transactivation and Fas/CD95-dependent apoptosis. Cell Death Differ 14: 411-421, 2007.

15. Zu K, Bihani T, Lin A, Park YM, Mori K and Ip C: Enhanced selenium effect on growth arrest by BiP/GRP78 knockdown in P53-null human prostate cancer cells. Oncogene 25: 546-554, 2006

16. Quick QA and Gewirtz DA: An accelerated senescence response to radiation in wild-type P53 glioblastoma multiforme cells. J Neurosurg 105: 111-118, 2006.

17. Xue W, Zender L, Miething C, Dickins RA, Hernando E, Krizhanovsky V, Cordon-Cardo C and Lowe SW: Senescence and tumour clearance is triggered by P53 restoration in murine liver carcinomas. Nature 445: 656-660, 2007.

18. Chen Z, Trotman LC, Shaffer D, Lin HK, Dotan ZA, Niki M, Koutcher JA, Scher HI, Ludwig T, Gerald W, Cordon-Cardo C and Pandolfi PP: Crucial role of P53-dependent cellular senescence in suppression of Pten-deficient tumorigenesis. Nature 436: 725-730, 2005.

19. Ventura A, Kirsch DG, McLaughlin ME, Tuveson DA, Grimm J, Lintault L, Newman J, Reczek EE, Weissleder R and Jacks T: Restoration of P53 function leads to tumour regression in vivo. Nature 445: 661-665, 2007.

20. Lin HK, Chen Z, Wang G, Nardella C, Lee SW, Chan CH, Yang WL, Wang J, Egia A, Nakayama KI, Cordon-Cardo C, Teruya-Feldstein J and Pandolfi PP: Skp2 targeting suppresses tumorigenesis by Arf-P53-independent cellular senescence. Nature 464: 374-379, 2010

21. Tchoghandjian A, Baeza N, Colin C, Cayre M, Metellus P, Beclin C, Ouafik L and Figarella-Branger D: A2B5 cells from human glioblastoma have cancer stem cell properties. Brain Pathol 20: 211-221, 2010.

22. Erdal H, Berndtsson M, Castro J, Brunk U, Shoshan MC and Linder S: Induction of lysosomal membrane permeabilization by compounds that activate P53-independent apoptosis. Proc Natl Acad Sci USA 102: 192-197, 2005

23. Garino C, Tomita T, Pietrancosta N, Laras Y, Rosas R, Herbette G, Maigret B, Quéléver G, Iwatsubo T and Kraus JL: Naphthyl and coumarinyl biarylpiperazine derivatives as highly potent human beta-secretase inhibitors. Design, synthesis, and enzymatic BACE-1 and cell assays. J Med Chem 49: 4275-4285, 2006.
24. Lefranc F, Facchini V and Kiss R: Proautophagic drugs: a novel means to combat apoptosis-resistant cancers, with a special emphasis on glioblastomas. Oncologist 12: 1395-1403, 2007.

25. Newlands ES, Stevens MF, Wedge SR, Wheelhouse RT and Brock C: Temozolomide: a review of its discovery, chemical properties, pre-clinical development and clinical trials. Cancer Treat Rev 23: 35-61, 1997.

26. Stupp R, Mason WP, van den Bent MJ, Weller M, Fisher B, Taphoorn MJ, Belanger K, Brandes AA, Marosi C, Bogdahn U, Curschmann J, Janzer RC, Ludwin SK, Gorlia T, Allgeier A, Lacombe D, Cairncross JG, Eisenhauer E and Mirimanoff RO: Radiotherapy plus concomitant and adjuvant temozolomide for glioblastoma. N Engl J Med 352: 987-996, 2005.

27. Kanzawa T, Germano IM, Komata T, Ito H, Kondo Y and Kondo S: Role of autophagy in temozolomide-induced cytotoxicity for malignant glioma cells. Cell Death Differ 11: 448-457, 2004.

28. Martinkova E, Maglott A, Leger DY, Bonnet D, Stiborova M, Takeda K, Martin S and Dontenwill M: alpha5beta1 integrin antagonists reduce chemotherapy-induced premature senescence and facilitate apoptosis in human glioblastoma cells. Int J Cancer 127: 1240-1248, 2010.

29. Guo GE, Ma LW, Jiang B, Yi J, Tong TJ and Wang WG: Hydrogen peroxide induces p16(INK4a) through an AUF1dependent manner. J Cell Biol 109: 1000-1005, 2010.

30. Wu Y, Zhang H, Dong Y, Park YM and Ip C: Endoplasmic reticulum stress signal mediators are targets of selenium action. Cancer Res 65: 9073-9079, 2005.

31. Linder S and Shoshan MC: Lysosomes and endoplasmic reticulum: targets for improved, selective anticancer therapy. Drug Resist Updat 8: 199-204, 2005.

32. Schönthal AH: Endoplasmic reticulum stress and autophagy as targets for cancer therapy. Cancer Lett 275: 163-169, 2009.

33. Kardosh A, Golden EB, Pyrko P, Uddin J, Hofman FM, Chen TC, Louie SG, Petasis NA and Schönthal AH: Aggravated endoplasmic reticulum stress as a basis for enhanced glioblastoma cell killing by bortezomib in combination with celecoxib or its non-coxib analogue, 2,5-dimethyl-celecoxib. Cancer Res 68: 843-851, 2008.

34. Chen TC, Lai KC, Yang JS, Liao CL, Hsia TC, Chen GW, Lin JJ, Lin HJ, Chiu TH, Tang YJ and Chung JG: Involvement of reactive oxygen species and caspase-dependent pathway in berberine-induced cell cycle arrest and apoptosis in C6 rat glioma cells. Int J Oncol 34: 1681-1690, 2009.

35. Salazar M, Carracedo A, Salanueva IJ, Hernández-Tiedra S, Lorente M, Egia A, Vázquez P, Blázquez C, Torres S, García S, Nowak J, Fimia GM, Piacentini M, Cecconi F, Pandolfi PP, González-Feria L, Iovanna JL, Guzmán M, Boya P and Velasco G: Cannabinoid action induces autophagy-mediated cell death through stimulation of ER stress in human glioma cells. J Clin Invest 119: 1359-1372, 2009.

36. Bursch W: The autophagosomal-lysosomal compartment in programmed cell death. Cell Death Differ 8: 569-581, 2001.

37. Bröker LE, Kruyt FA and Giaccone G: Cell death independent of caspases: a review. Clin Cancer Res 11: 3155-3162, 2005. 